\title{
Structural analysis of an Octameric Resorcinarene Self-assembly in Toluene and its Morphological Transition by Temperature
}

Shota Fujii* and Kazuo Sakurai*

Department of Chemistry and Biochemistry, University of Kitakyushu, 1-1 Hibikino, Kitakyushu,

Fukuoka 808-0135, Japan

Table of Contents

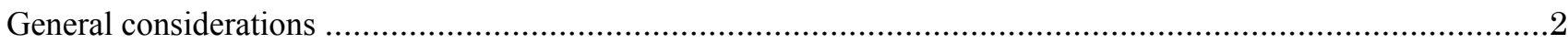

Characterization of the structure of the resorcinarene self-assembly ........................................................2

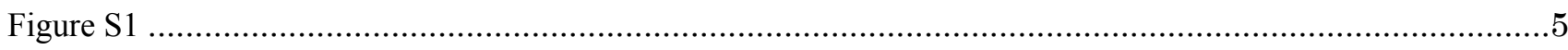

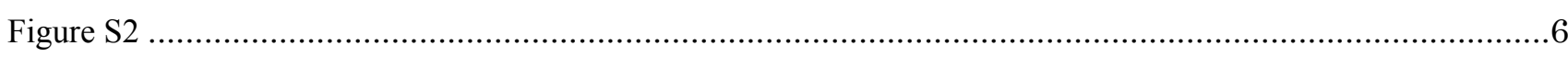

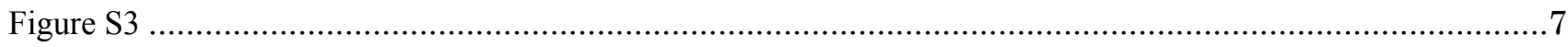

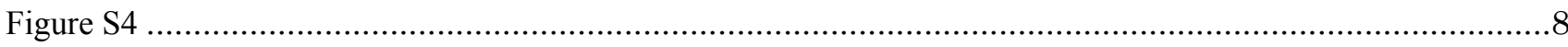

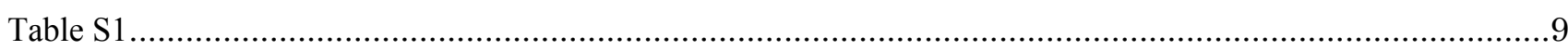


General considerations

Materials. $C$-undecylresorcinarene was purchased from Sigma Aldrich Co., used without further purification. The other chemical reagents were purchased from Tokyo Chemical Industry Co., Tokyo Japan.

Sample preparation. The powder of $C$-undecylresorcinarene was dispersed in toluene to be required concentration $(10 \mathrm{mg} / \mathrm{mL}$ is normal concentration for all measurements). Gentle heating and agitation for the dispersed solution give a clear solution. The prepared samples were left for at least one day to equilibrate at room temperature.

Characterization of the structure of the resorcinarene self-assembly

Small angle X-ray scattering (SAXS) measurements. SAXS measurements were carried out at the BL40B2 beamline of the SPring-8 facility, Hyōgo Prefecture, Japan. A digital detector (Pilatus-100K) was placed $1 \mathrm{~m}$ from the sample. The wavelength of the incident beam $(\lambda)$ was adjusted to $0.071 \mathrm{~nm}$. This setup provided a $q$ range of $0.20-10 \mathrm{~nm}^{-1}$, where $q$ is the magnitude of the scattering vector, defined as $q=4 \pi \sin \theta / \lambda$, with a scattering angle of $2 \theta$. The X-ray transmittance of the samples was determined by using ion chambers located in front of and behind the sample. The detailed experimental procedures are reported elsewhere. ${ }^{1}$ The absolute SAXS intensities were recorded using the absolute scattering intensities of water. ${ }^{2,3}$

Determination of the resorcinarene self-assembly structure from SAXS profiles. For the fitting of the SAXS profiles of the resorcinarene self-assembly (nanoparticle with 8 resorcinarenes: $\mathbf{N R}_{\mathbf{8}}$ ), we employed the model of a core-shell spherical [Eq. (1)] and a finite core-shell cylinder [Eq. (2)-(3)] model described by the following expression:

$$
I(q)=\left\{3 V_{\mathrm{C}}\left(\rho_{\mathrm{C}}-\rho_{\mathrm{S}}\right) \frac{j_{1}\left(q R_{\mathrm{C}}\right)}{q R_{\mathrm{C}}}+3 V_{\mathrm{S}} \rho_{\mathrm{S}} \frac{j_{1}\left(q R_{\mathrm{S}}\right)}{q R_{\mathrm{S}}}\right\}^{2}
$$




$$
\begin{gathered}
I(q)=\frac{N}{V_{\mathrm{S}}} \int_{0}^{\pi / 2} f^{2}(q, \alpha) \sin \alpha d \alpha \\
f(q, \alpha)=2\left(\rho_{\mathrm{C}}-\rho_{\mathrm{S}}\right) V_{\mathrm{C}} j_{0}(q H \cos \alpha) \frac{J_{1}\left(q R_{\mathrm{C}} \sin \alpha\right)}{\left(q R_{\mathrm{C}} \sin \alpha\right)}+2\left(\rho_{\mathrm{S}}-\rho_{\mathrm{Sol}}\right) V_{\mathrm{S}} j_{0}[q(H+t) \cos \alpha] \frac{J_{1}\left[q\left(R_{\mathrm{C}}+t\right) \sin \alpha\right]}{\left[q\left(R_{\mathrm{C}}+t\right) \sin \alpha\right]}
\end{gathered}
$$

Here, $R_{\mathrm{c}}, R_{\mathrm{s}}, H$, and $t$ are the outer radii of the cross-sectional core and particle (core + shell), half values of the cylinder length, and thickness of the shell, respectively. $\rho_{\mathrm{c}}, \rho_{\mathrm{s}}$, and $\rho_{\mathrm{sol}}$ are the electron density $\left(\mathrm{e} \mathrm{nm}^{-3}\right)$ of the core, the shell, and the solvent, respectively. $J_{1}, j_{0}$, and $j_{1}$ are the first Bessel function and first and second spherical Bessel function, respectively. $V_{\mathrm{C}}$ and $V_{\mathrm{S}}$ are the particle volume of the core and particle (core + shell), respectively. The SAXS profiles in the low $q$ region follow the Guinier relation given by the following equation: ${ }^{6}$

$$
I(q)=I(0) \exp \left(-q^{2} R_{\mathrm{g}}^{2} / 3\right)
$$

where $I(0)$ is the forward scattering intensity at $q=0 . I(0)$ and the gyration radius $\left(R_{\mathrm{g}}\right)$ are determined from the intercept and the slope of the $\ln (I(q))$ vs. $q^{2}$ plot (Guinier plot). Due to inter-particle interference, the $I(0)$ and $R_{\mathrm{g}}$ values depend on the sample concentration. In order to remove the concentration effects, the SAXS intensities recorded at different concentrations were extrapolated to zero concentration.

Determination of micellar molar mass by SAXS. The molar mass of the micelles can be given by the following equation:

$$
\begin{gathered}
M_{w}=\lim _{c \rightarrow 0} \frac{I(0)}{K_{e} c} \#(4) \\
K_{e}=N_{\mathrm{A}}\left(r_{\mathrm{e}} \gamma_{\mathrm{ave}}\right)^{2} \#(5) \\
\gamma_{\text {ave }}=\frac{n_{\mathrm{e}, \text { solute }}}{M_{\text {solute }}}-\bar{v} \frac{\rho_{\text {sol }} n_{\mathrm{e}, \text { solvent }}}{M_{\text {solvent }}} \#(6)
\end{gathered}
$$

where $M_{\mathrm{w}}$ is weight averaged molecular weight, $c$ is the solute concentration. $K_{\mathrm{e}}$ is optical constant in SAXS and described by eq. (5). $N_{\mathrm{A}}$ and $r_{\mathrm{e}}$ are Avogadro's number and classical electron radius, respectively. $\gamma_{\text {ave }}$ is described by eq. (6), where $n_{\mathrm{e}, \text { solute }}, n_{\mathrm{e}, \text { solvent }}, M_{\text {solute }}$, and $M_{\text {solvent }}$, respectively. The 
term $\bar{v}$ indicates the specific volume of $\mathbf{N R}_{\mathbf{8}}$ in the solution, which can be determined by the density of the sample solutions and the solvent. 
Figure S1

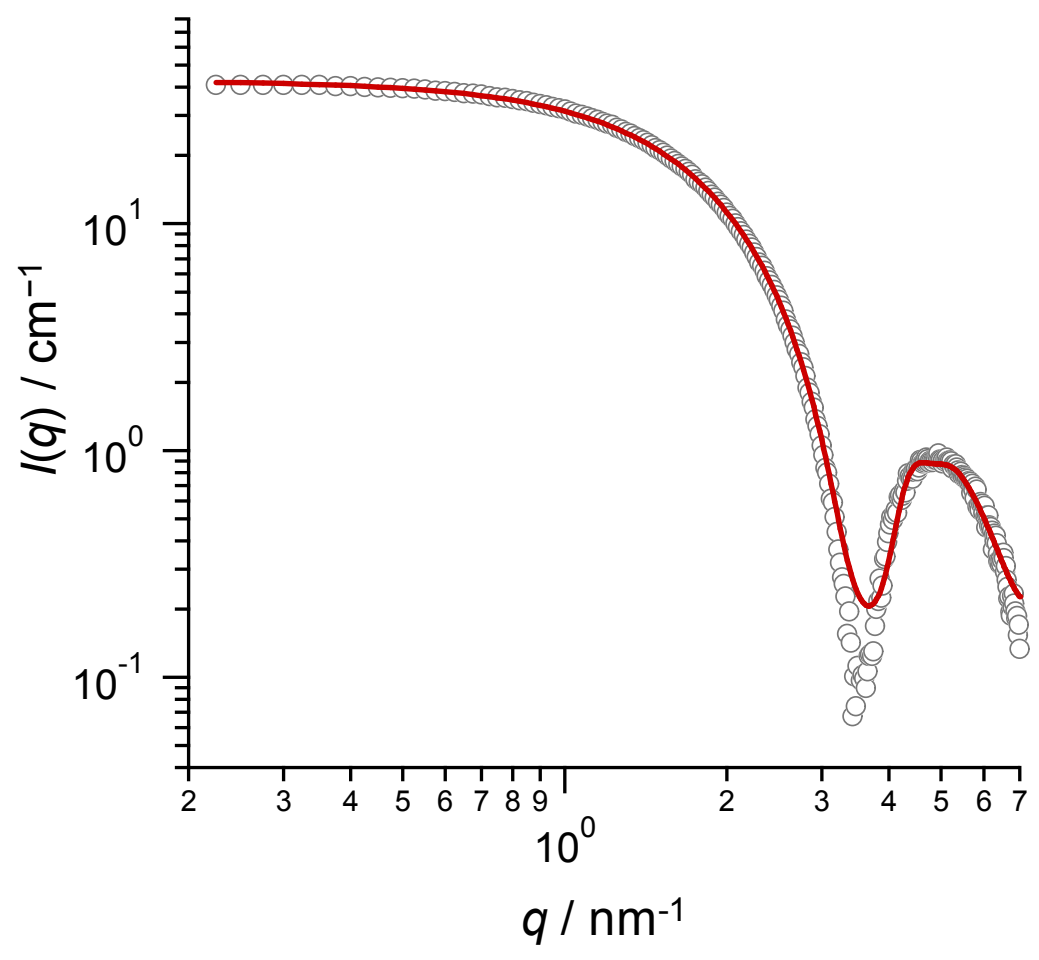

Figure S1. Fitting curve (red curve) for $\mathbf{N R}_{\mathbf{8}}$ in toluene calculated from the dummy atom assembly prepared with the program DAMMIN. 
Figure S2

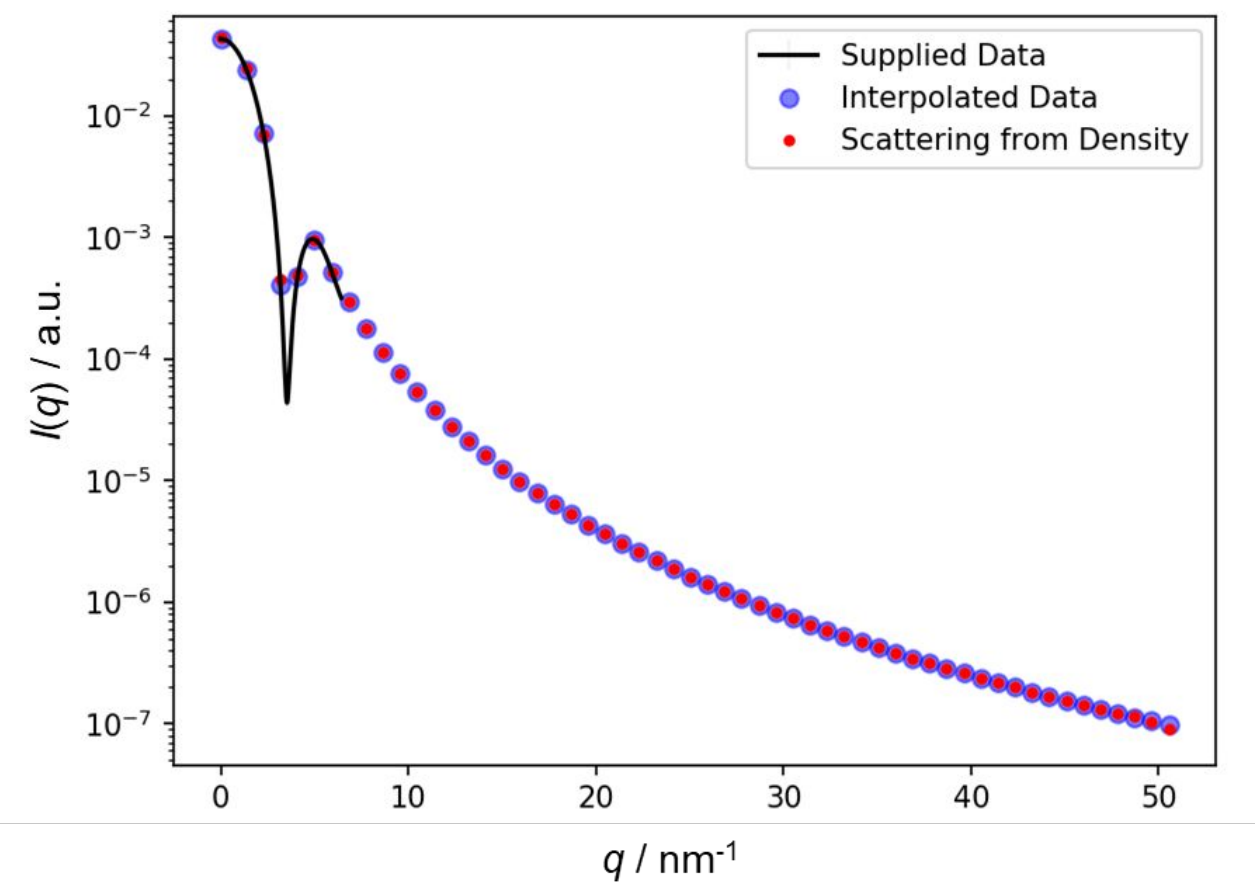

Figure S2. Fitting curve (red curve) for $\mathbf{N R}_{\mathbf{8}}$ in toluene calculated from the electron density map prepared with the program DENSS. 
Figure S3

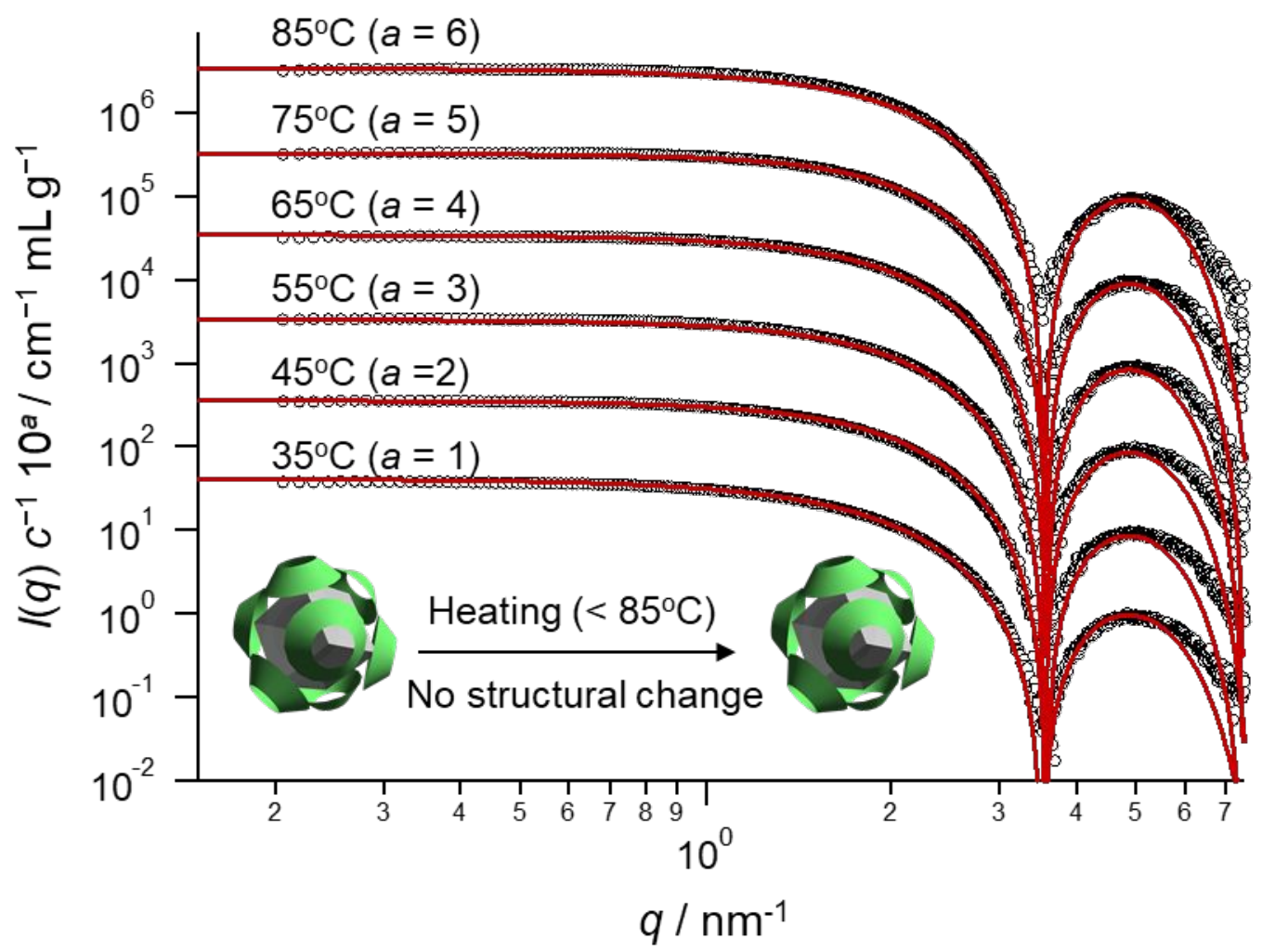

Figure S3. SAXS profiles of $\mathbf{N R}_{\mathbf{8}}$ in toluene at various temperatures. The profiles at temperatures higher than this $\left(35-85^{\circ} \mathrm{C}\right)$ are shown here. The red curves were calculated using core-shell spherical models. 

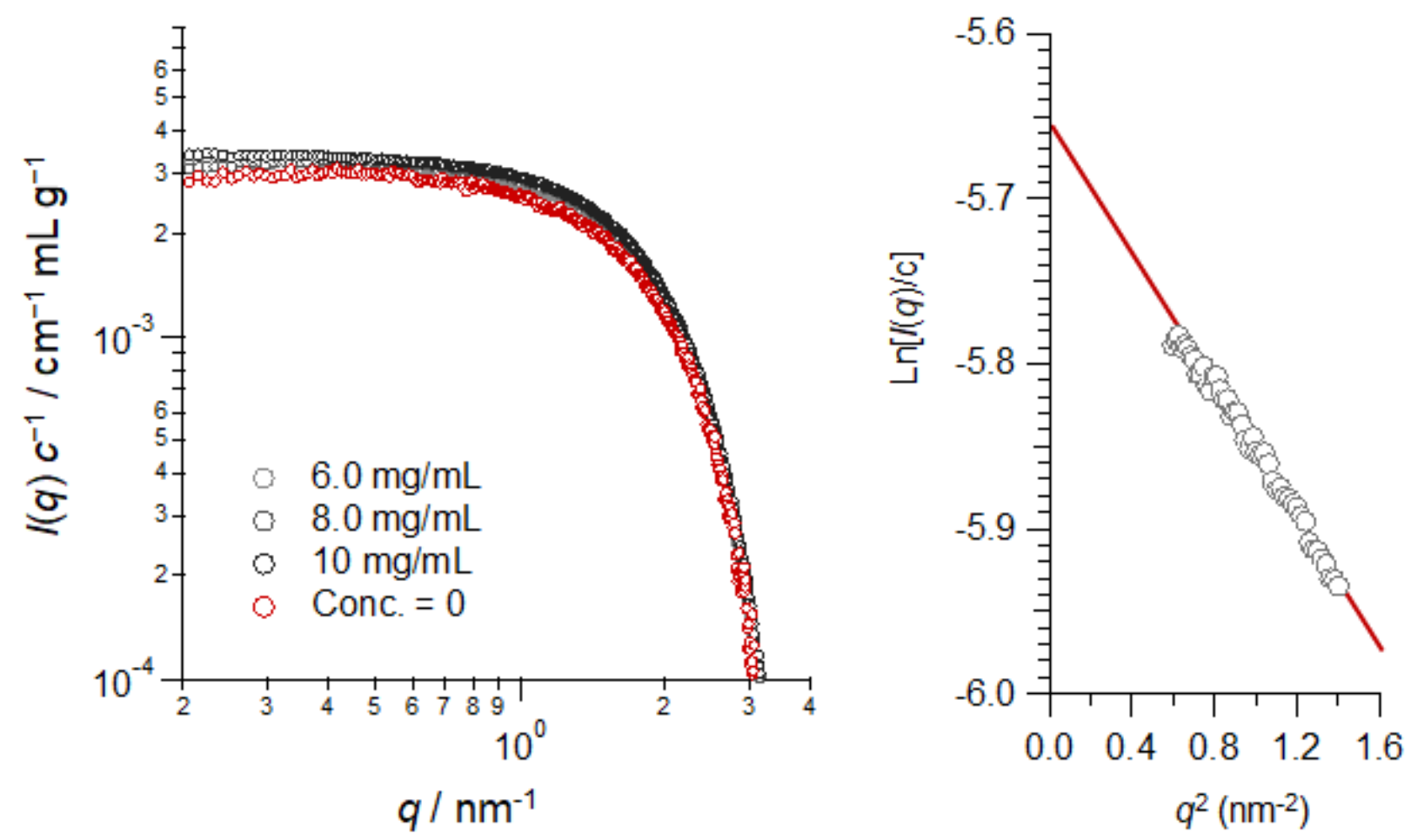

Figure S4. (Left side) $I(q) / c$ as a function of $q$ for $\mathbf{N R}_{\mathbf{8}}$ in toluene at different concentrations (open gray circles) at $85^{\circ} \mathrm{C}$; the extrapolated values at infinite dilution for each $q$ are shown by red circles. (Right side) Guinier plots [i.e., $\ln I(q) / c$ versus $q^{2}$ ] constructed from the extrapolated intensities. We determined the molar mass of the scatterer from the intercept value using equation $(4) \sim(6)$. 
Table S1

Table S1. SAXS Fitting Parameters and Gyration Radius $\left(R_{\mathrm{g}}\right)$ for C-undecylresorcinarene Capsules in Toluene at Different Temperatures

\begin{tabular}{|c|c|c|c|c|c|c|c|c|}
\hline $\begin{array}{c}\text { Temp. } \\
{\left[{ }^{\circ} \mathrm{C}\right]}\end{array}$ & $\begin{array}{c}R_{\mathrm{c}, \mathrm{sphere}} \\
{[\mathrm{nm}]}\end{array}$ & $\begin{array}{c}R_{\mathrm{s}, \mathrm{sphere}} \\
{[\mathrm{nm}]}\end{array}$ & $\begin{array}{c}R_{\mathrm{c}, \mathrm{cylinder}} \\
{[\mathrm{nm}]}\end{array}$ & $\begin{array}{c}R_{\mathrm{s}, \text { cylinder }} \\
\text { [nm] }\end{array}$ & $\begin{array}{c}\text { Length } \\
{[\mathrm{nm}]}\end{array}$ & $\begin{array}{c}\rho_{\mathrm{c}} \\
{\left[\mathrm{e} \mathrm{nm}^{-3}\right]}\end{array}$ & $\begin{array}{c}\rho_{\mathrm{s}} \\
{\left[\mathrm{e} \mathrm{nm}^{-3}\right]}\end{array}$ & $\begin{array}{c}R_{\mathrm{g}} \\
{[\mathrm{nm}]}\end{array}$ \\
\hline 15 & 0.65 & 1.11 & 0.65 & 1.11 & 3.2 & 282 & 278 & 1.04 \\
\hline 5 & 0.65 & 1.11 & 0.65 & 1.11 & 3.5 & 282 & 278 & 1.16 \\
\hline-5 & 0.65 & 1.11 & 0.65 & 1.11 & 4.5 & 282 & 278 & 1.38 \\
\hline-15 & 0.65 & 1.11 & 0.65 & 1.11 & 6.0 & 282 & 278 & 1.72 \\
\hline-25 & 0.65 & 1.11 & 0.65 & 1.11 & 8.5 & 282 & 278 & 2.16 \\
\hline
\end{tabular}

